\title{
CFD analysis of asymmetric mixing at different inlet configurations of a split-and-recombine micro mixer
}

\author{
Torben Frey ${ }^{1}$ - Rieke Schlütemann ${ }^{1}$ - Sebastian Schwarz ${ }^{2}$. Philip Biessey ${ }^{2}$ Marko Hoffmann ${ }^{1}$. \\ Marcus Grünewald ${ }^{2} \cdot$ Michael Schlüter ${ }^{1}$
}

Received: 18 February 2021 / Accepted: 10 May 2021 / Published online: 1 July 2021

(C) The Author(s) 2021

\begin{abstract}
In the scope of the ENPRO II initiative (Energy Efficiency and Process Intensification for the Chemical Industry), a major challenge of process intensification of polymer synthesis in continuous systems is fouling. Pre-mixing is a key aspect to prevent fouling and is achieved through milli and micro structured devices (Bayer et al. 2000). While equal volume flow ratios are well investigated in milli and micro systems, asymmetric mixing tasks have received less attention. This paper investigates the dependency of mixing phenomena on different flow rate ratios and modified inlet geometries. A split-andrecombine (SAR) mixer is modified by means of an injection capillary to facilitate the asymmetric mixing task. Asymmetric volume flows of ratios between 1:15 and 1:60 are investigated; the velocity ratios range from 0.5 to 2 . The setup is simulated with the Computational Fluid Dynamics (CFD) tool ANSYS ${ }^{\circledR}$ FLUENT. The species equation is solved directly without the use of micro mixing models. The simulation is validated by means of a concentration field in a mixing Tee using LaserInduced Fluorescence (LIF) with a Confocal Laser Scanning Microscope (CLSM). The three dimensional flow structures and the mixing quality are analyzed as a measure for micro mixing. The calculated concentration fields show good agreement with the experimental results and reveal the secondary flow structures and chaotic advection within the channel. The injection of the small feed stream is found to be very efficient when drawn into the secondary structures, increasing the potential of diffusive mixing. CFD simulations help to understand and locate such structures and improve the mixing performance.
\end{abstract}

Keywords Micro mixing Computational fluid dynamics · Potential for diffusive mixing · Asymmetric flow rate ratios (FRR)

\section{Introduction}

In the last two decades, micro systems have enabled the creation of polymers with tailored properties, including highly complicated block- and copolymer structures in the lab [2-6]. Deep knowledge about reaction mechanisms, enhanced control of temperature and reaction time, and sophisticated analytics allow the precise design of polymer chain lengths and molecular weight distributions in such

Marko Hoffmann

marko.hoffmann@tuhh.de

1 Institute of Multiphase Flows, Hamburg University of Technology, Hamburg, Germany

2 Laboratory of Fluid Separations, Ruhr-University Bochum, Bochum, Germany micro systems. Recent data driven approaches have proven the feasibility of transferring complex polymers directly from the notepad into the lab [7,8], yielding endless possibilities to polymer design.

While these concepts work well in the lab, industry applications are interested in scale-up and handling. The group around Yoshida has worked on several studies to enhance throughput in capillary reactors using the numbering up philosophy [9-11]. The group achieved a service time of several hours, limited by fouling and the consequent increasing pressure drop. For production processes a strategy to prevent or reduce fouling is necessary. One drawback of capillary micro reactors is the challenge of cleaning in place due to the small dimensions. Established industrial applications tend to prefer milli scale equipment, where the temperature control is still sufficient. The residence time distribution is kept narrow by static mixing elements generating additional radial dispersion. To ensure the homogeneity of reagents in 
milli systems, an upstream micro mixer can be used. The fouling reducing effect of pre-mixing was shown by Bayer et al. [1] in a milli scale process and is well-known for micro systems $[12,13]$.

Such a pre-mixing stage combines the respective monomers and initiators for the polymer reactions. A large volume flow of monomer is mixed with a small amount of initiator. For this purpose, an off-the-shelf split-and-recombine (SAR) mixer by Ehrfeld Mikrotechnik $\mathrm{GmbH}$ is modified with a capillary. The mixer module is shown in Fig. 1a. The modification ensures small velocity ratios to prevent areas of recirculation. The complex interaction of reaction rate, residence time and micro mixing has an impact on the product and potential fouling within the pre-mixer.

In this work, a case study is carried out to investigate the dependency of micro-mixing on flow rate ratios and inlet configuration of a pre-mixing stage. Understanding the mixing phenomena is essential for design processes with uniform reaction progress and the minimization of fouling. In addition to the non-reactive mixing study, a reactive system is simulated in the same geometry to link the observed mixing phenomena to the selectivity of the chemical reactions.

\section{Theory}

\section{Transport Mechanisms in Micro Systems}

To understand the transport phenomena in milli and micro systems, it is important to look at the flow regime. Due to the small dimensions of micro systems, the REYNOLDS number

$R e=\frac{U d}{v}$

typically is small (e.g. $R e<100$ ). For flow channels $U$ is the mean flow velocity, $d$ is the hydraulic diameter, and $v$ is the kinematic viscosity of the fluid. The flow in these systems is typically laminar, meaning the Lagrangian trajectories of the fluid parcels are equal to the streamlines of the velocity field. Mass transport perpendicular to the streamlines is therefore dominated by molecular diffusion. The ratio of momentum dissipation and molecular diffusion is given by the SCHMIDT number

$S c=\frac{v}{D_{i j}}$

where $D_{i j}$ is the binary diffusion coefficient of the two fluids $i$ and $j$. A diffusion dominated flow is prevalent when the radial PÉCLET number

$P e_{\mathrm{r}}=R e \cdot S c=\frac{U d}{D_{i j}}$ is equal to or smaller than one. The PÉCLET number gives the ratio of advective and diffusive mixing. Mixing in diffusion dominated flows relys on small diffusion lengths, chaotic advective effects, and secondary flows.

Diffusion Length The speed of molecular diffusion is determined by the diffusion coefficient $D_{i j}$ and depends on physical properties of solvent/solute and temperature. Due to prescribed process parameters, the diffusion speed can usually not be influenced. In order to minimize mixing time, reduction of the diffusion length is the main objective in laminar flow systems [14]. Beside heat transfer, this is the main benefit of miniaturization and will however lead to an increase in pressure drop. A trade-off between miniaturization and pressure drop is found in milli systems. Laminating the flow is an additional measure to reduce the diffusion path within a mixing device. A SAR mixer uses serial lamination to create $2^{n+1}$ lamellae, with $n$ being the number of SAR stages. The principle of serial lamination is shown in Fig. $1 b$.

Chaotic Advection Despite the steady laminar flow regime in micro channels, the streamlines can flow across each other which leads to a change of the Lagrangian trajectories. This phenomenon is called chaotic advection. It occurs due to disruptions in laminar flow and is different from turbulence in a way that the velocity field is constant over time, whereas in turbulent flow the velocity fluctuates at random [15]. Chaotic advection can be induced passively by geometrical obstacles, as demonstrated by Stroock et al. by the use of herringbone mixers in STOKES flow applications [16]. The chaotic advection in mixing Tees was extensively investigated by Kockmann [17]. His group documented the transition from a steady engulfment flow at $130<R e<$ 240 to a time-periodic pulsation of the flow pattern before it develops a turbulent characteristic at $R e>500$. Chaotic advection is expected to occur in the SAR mixer to a certain extend, especially when increasing the REYNOLDS number.

Secondary Flows Further reduction of mixing time can be achieved by increasing the potential of diffusive mixing. The potential $\Phi$ can be expressed as the volume specific contact area of two miscible fluids [18]. Secondary flows are beneficial for increasing the potential and can be induced in form of engulfment flow or DEAN flow for example. Both phenomena occur due to inertial forces of the fluid. In these kinds of stationary vortices, the contact area of the fluids is largely increased. However, axial vortices or dead zones broaden the residence time distribution and may have negative effects on the mixing time while creating a mixing barrier to the rest of the flow structure. Reis et al. [19] use secondary flows within a segmented droplet flow to achieve a residence time distribution (RTD) similar to plug flows 
Fig. 1 a Split-and-recombine mixer by Ehrfeld Mikrotechnik $\mathrm{GmbH}$ modular micro reaction system (MMRS); b serial lamination scheme of a typical SAR mixer for flow rate ratio $\mathrm{FRR}=1$ (a)

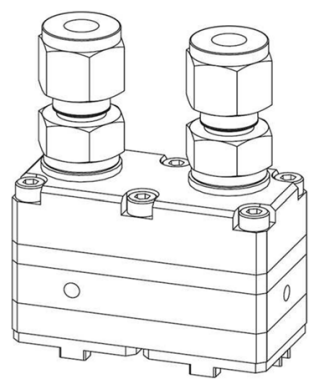

(b)

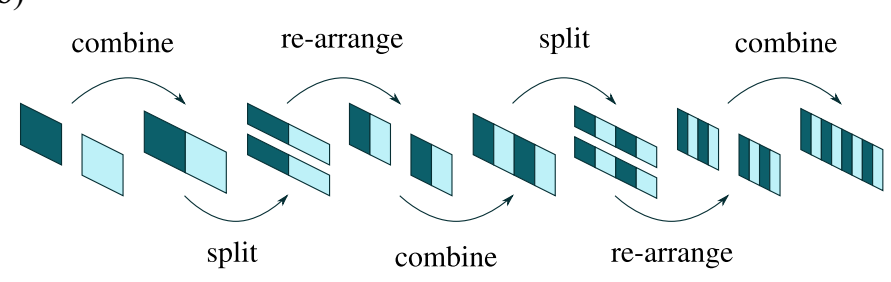

and rapid mixing. To avoid axial recirculation, the velocitiy ratios of injection and channel are kept between 0.5 and 2 .

\section{Reactive Mixing}

Chemical reactions take place at a molecular level and therefore micro mixing on a molecular level is a precondition. The FOURIER number for mass transfer by diffusion

$F o=\frac{t_{\mathrm{r}}}{t_{\mathrm{m}}}=\frac{l D_{i j}}{U d^{2}}$

relates residence time $t_{\mathrm{r}}$ to the mixing time $t_{\mathrm{m}}$. It is used to predict whether the reaction rate is limited by diffusion and micro mixing. According to DeMello et al. [20], a high FOURIER number indicates a chemical regime where the reaction constant can be neglected. However, when the residence time is small compared to the mixing time, the reaction constant is important and mixing affects the reaction in the so-called diffusional regime.

Following the case study on mixing phenomena the esterification of di-isocyanate is investigated as an exemplary reactive system. Di-isocyanates are a common monomer for anionic polymerization. In the ENPRO II initiative, the diisocyanate is mixed with a base as initiator solved in an alcohol solution. The esterification of the isocyanate groups with alcohol is an unwanted side reaction. The given mixing problem is allocated in the diffusional regime with a low FOURIER number. The reactions can be simplified to a competitive-consecutive reaction scheme

$\mathrm{A}+\mathrm{B} \stackrel{k_{1}}{\longrightarrow} \mathrm{C}$ and
$\mathrm{C}+\mathrm{B} \stackrel{k_{2}}{\longrightarrow} \mathrm{D}$.

The alcohol $B$ is added to the first isocyanate group of reagent $A$ to form a mono-urethane $C$. In the consecutive reaction, the second isocyanate of intermediate $C$ then reacts with alcohol $B$ to form a di-urethane $D$. The viscosity increases with molecular weight. The alcohol $B$ is the limiting component of the reaction scheme due to its under supply. The reaction rate depends on the available isocyanate groups $\left(k_{1}>k_{2}\right)$ and consequently, di-urethane $D$ will scarcely form if the reagents are mixed perfectly. The formation is only possible if there is a local substoichiometric supply of di-isocyanate $A$ due to insufficient mixing. The formation of di-urethane $D$ and the consequent increase in viscosity is assumed to be a product of a wide RTD and an insufficient mixing time which is larger than the reaction rate.

\section{Methods and Setup}

\section{Model Setup}

The reactive mixing model is simulated with the commercial CFD software ANSYS ${ }^{\circledR}$ FLUENT v18.2 (Finite Volume Method, FVM). The two reagents are mixed inside a SAR mixer by Ehrfeld Mikrotechnik GmbH, see Fig. 1a. The mixer is used to enable fast mixing. To facilitate the large flow rate ratios (FRR) with low velocity ratios, a 1/32" capillary is inserted into one inlet of the SAR mixer. The di-isocyanate enters the mixer through inlet 1 and 2 - the alcohol is injected through the capillary. The position of the capillary is modified in the mixer inlet as illustrated in Fig. 2.

An overview of the model setup is given in Table 1. Due to the low REYNOLDS number within the mixing structure $\left(20<R e_{\mathrm{SAR}}<80\right)$ a stationary-laminar model is chosen to be appropriate for the simulation. The species transport model uses the STEFAN-MAXWELL equations and is solved directly without the use of micro mixing models. The governing equations are to be found in the Supplementary Info. To ensure grid independence for the species equation, the grid size is refined locally. Bothe et al. [21] derived a correlation of the smallest concentration length scale $\lambda_{\text {conc }}$ for laminar, stationary flow regimes

$\lambda_{\text {conc }}=\frac{\lambda_{\mathrm{vel}}}{\sqrt{S c}}=\frac{v}{U \sqrt{S c}}$

from the BATCHELOR length scale. In (6), $\lambda_{\text {vel }}$ is the smallest velocity length scale, $v$ is the kinematic viscosity, and $U$ is the characteristic velocity. In this case setup, $\lambda_{\text {conc }}$ is in the range of $10 \mu \mathrm{m}$.

The reaction is slow and will not be complete at the end of the domain. The mixture can therefore be regarded as a solution with the di-isocyanate as solvent and the products 

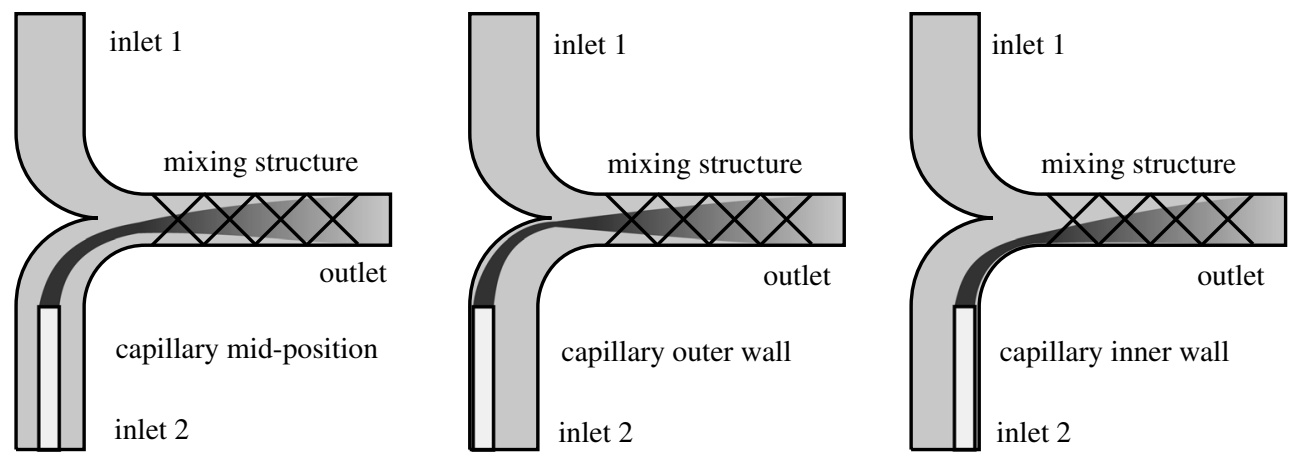

Fig. 2 Geometry scheme: Modified inlet of a SAR mixer by Ehrfeld Mikrotechnik GmbH, capillary in mid-channel position, in outer and inner wall position

as solute. The viscosity of the solution is calculated by the solute-solvent approach

$\eta_{\text {sol }}=\eta_{\text {solvent }}\left(1+\eta_{\mathrm{sp}}\right)$

with dimensionless specific viscosity of the solute $\eta_{\mathrm{sp}}$ and the solvent viscosity $\eta_{\text {solvent }}[22]$.

The simulations are carried out at three different FRR, ranging from 14 to 56 . Together with the capillary position variation the parameter study yield the operating conditions listed in Table 2.

\section{Experimental Setup}

The species transport model is validated via the concentration field. A 3D-concentration field is measured by a Confocal Laser Scanning Microscope (CLSM). Due to the stationary nature of the flow, the CLSM is able to record the Laser-Induced Fluorescence (LIF) at high spatial resolutions in the range of a few micrometers. Rhodamine B is used as a passive tracer in highly diluted form. Rhodamine B has an absorption maximum at $555 \mathrm{~nm}$ and an emission peak at $580 \mathrm{~nm}$ [23]. The intensity of emission correlates linearly with the Rhodamine B concentration at a set temperature and allows easy calibration [14].

The experimental setup with a mixing Tee are shown in Fig. 3. DI-water and Rhodamine B $(1 \mu \mathrm{M})$ solution is supplied in two enclosed containers. The liquid media is driven by pressurized air to ensure pulsation free flow. Two thermal mass flow controllers (LIQUI-FLOW from Bronkhorst Deutschland Nord GmbH, FC-02, FC-03) and one coriolis type mass flow controller (CORI-FLOW from Bronkhorst Deutschland Nord GmbH, FC-01) are used to set the mass flow rates for the DI-water and the Rhodamine B solution. For the experimental validation, a mixing Tee with no internal mixing structure is fed from both sides with DI-water. Rhodamine B solution is injected by a capillary, as illustrated in Fig. 3 bottom left. The mixing Tee has a rectangular cross section with a channel height of $1.6 \mathrm{~mm}$, and the 1/32" injection capillary has an inner diameter of $500 \mu \mathrm{m}$.

The mixing Tee has a transparent lid which grants optical access to the CLSM. The OLYMPUS FV1000 system is used as a CLSM system. The Ar-laser produces an excitation wavelength of $488 \mathrm{~nm}$. The mixing Tee is placed on a traverse and can be moved to adjust the field of view of the microscope. Three fields of view (see Fig. 4) are recorded: Directly behind the capillary, at the mixer bend, and just after the two DI-water streams make contact. To ensure a stationary flow, the flow rates are set to achieve Reynolds numbers between 30 and 120 within the channel. Three different flow rate ratios (FRR) are set for validation. The operating conditions of all experiments are displayed in Table 3.

Table 1 CFD simulation: Model Setup

\begin{tabular}{lllll}
\hline Fluid system & Solver & Inlet conditions & Walls & Outlet \\
\hline Water-Rhodamine B & pressure-based & Velocity Inlet (Table 3) & No Slip & Outflow \\
Di-Isocyanate-Alcohol & pressure-based & Velocity Inlet (Table 2) & No Slip & Outflow \\
& Species & Reaction & Schmidt number & Grid Size \\
Fluid System & Binary Diffusion & none & $S c=3600 \quad>1.2 \mu \mathrm{m}$ \\
Water-Rhodamine B & Binary Diffusion & none & $1600<S c<8000 \quad>4.8 \mu \mathrm{m}$ \\
Di-Isocyanate-Alcohol & Multicomponent & Finite Rate & $1600<S c<8000$ \\
Di-Isocyanate-Alcohol & & &
\end{tabular}


Table 2 CFD simulation: Operating parameters for the reactive mixing in the SAR mixer

\begin{tabular}{|c|c|c|c|c|c|}
\hline $\begin{array}{l}\text { Capillary } \\
\text { Position }\end{array}$ & $\begin{array}{l}\text { Flow Rate } \\
\text { Di-isocyanate } \\
{[\mathrm{mL} / \mathrm{h}]}\end{array}$ & $\begin{array}{l}\text { Flow Rate } \\
\text { Alcohol } \\
{[\mathrm{mL} / \mathrm{h}]}\end{array}$ & $\operatorname{Re}_{\mathrm{SAR}}$ & $\begin{array}{l}\text { Flow Rate } \\
\text { Ratio } \\
{[-]}\end{array}$ & $\begin{array}{l}\text { Operating } \\
\text { Temperature } \\
{\left[{ }^{\circ} \mathrm{C}\right]}\end{array}$ \\
\hline Mid-channel & 132 & 9.4 & 20 & 14 & 25 \\
\hline Mid-channel & 163 & 9.4 & 40 & 28 & 25 \\
\hline Mid-channel & 526 & 9.4 & 80 & 56 & 25 \\
\hline Outer wall & 132 & 9.4 & 20 & 14 & 25 \\
\hline Outer wall & 263 & 9.4 & 40 & 28 & 25 \\
\hline Outer wall & 526 & 9.4 & 80 & 56 & 25 \\
\hline Inner wall & 132 & 9.4 & 20 & 14 & 25 \\
\hline Inner wall & 263 & 9.4 & 40 & 28 & 25 \\
\hline Inner wall & 526 & 9.4 & 80 & 56 & 25 \\
\hline
\end{tabular}

\section{Results and Discussion}

\section{Non-Reactive Species Transport}

Prior to discussing the results of the reactive flow, the nonreactive model is validated via the concentration field distribution. In the water-Rhodamine B system, the fluorescence intensity is correlated linearly with the Rhodamine B concentration by measuring the intensity of stepwise diluted solutions. The OLYMPUS FV 1000 records greyscale values: A value of zero (black) corresponds to DI-water, the greyscale value for $1 \mu \mathrm{M}$ Rhodamine B solution is normalized to 1 (white). By creating a stack of 2D-plane images, the CLSM allows to interpolate a 3D image of the concentration distribution within the mixing Tee, as shown in Fig. 4a. Three fields of view are recorded, one directly after the injection position, one in the bend of the Tee and one after the joining of the two DI-water streams. For analysis, three cross sections of these 3D images are used, as shown in $4 b$ for example.

The concentration field of the cross sections is analyzed by means of the potential for diffusive mixing $\Phi$, and mixing quality $\alpha$. For the latter, Sommer et al. [24] give a statistical approach to quantifying the homogeneity of a mixture. The mixing quality

$\alpha=1-\sqrt{\frac{\sigma^{2}}{\sigma_{\max }^{2}}}$

of the non-reactive flow evaluates the variance of the mass flow weighted concentration distribution $\sigma$ at different cross sections normal to the flow direction, according to Kockmann [17]. Since the overlying velocity field is not recorded, the mixing quality $\alpha$ can only give a qualitative comparison between measurements. Therefore, the mixing quality is evaluated for the SAR mixer results only. The following
Fig. 3 Experimental setup of LIF measurements in mixing Tee: Fluorescence (Rhodamine B) solution is injected into a capillary modified inlet of the mixing Tee

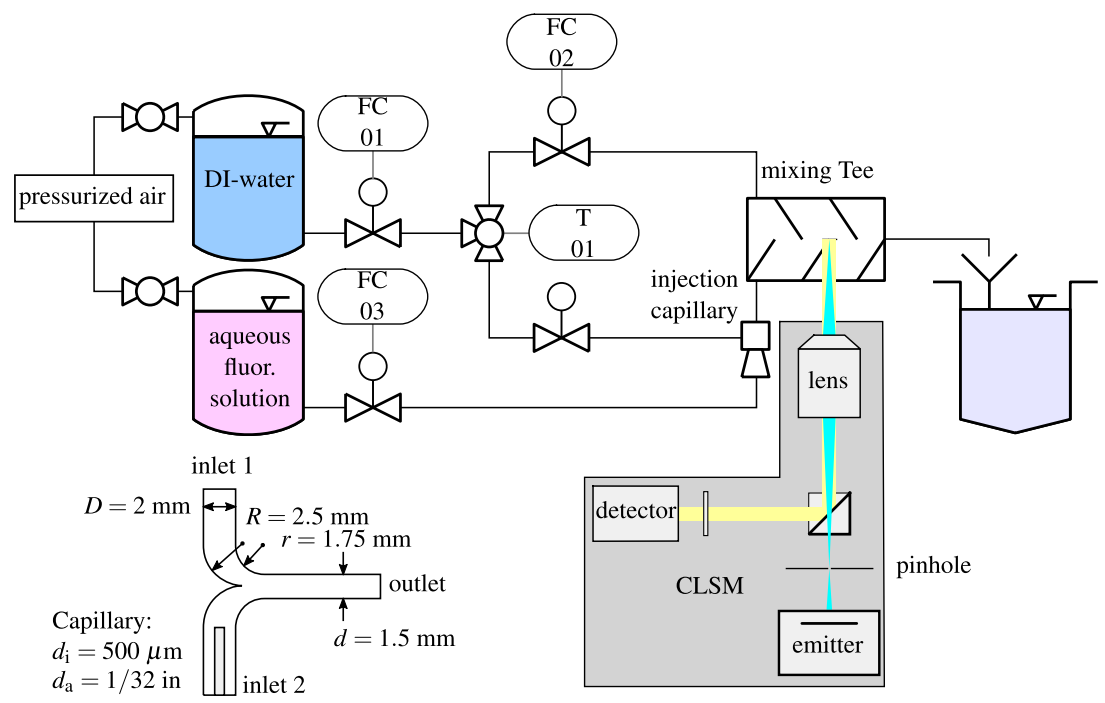


Fig. 4 a 3D images recorded with the CLSM, illustrated in the mixing Tee with injection capillary: DI-water in black, Rhodamine B solution in white. $R e_{\text {channel }}=60, \mathrm{FRR}=30 ; \mathbf{b}$ Field of View 3: Cross section image interpolated from stack of 2D-planes with resolution and size information; $\mathbf{c}$ Field of View 3: Detection of contact area boundary, perimeter $P$, enclosed area $A$, sphericity $\Psi$, and potential for diffusive mixing $\Phi$; d Cross section images of fields of view 1,2, and 3 for different capillary positions - Experimental results vs CFD results including radial velocity vectors

Table 3 Operating parameters for the validation measurement series (a) Field of View 1

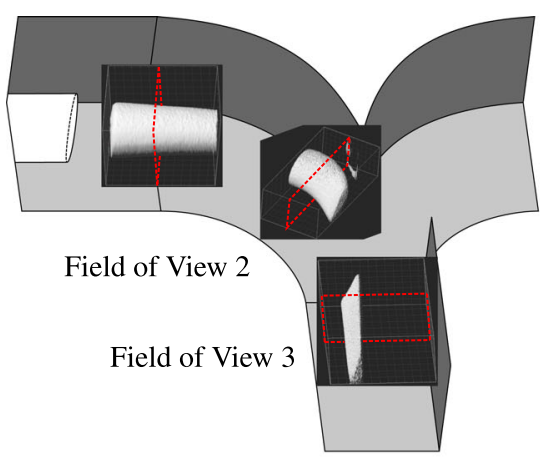

(b)

(c)

interpolated cross section image boundary detection
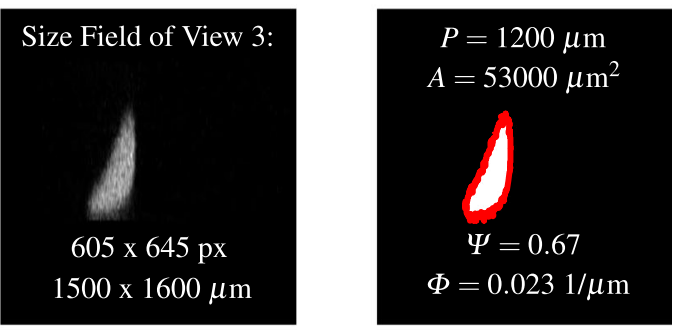

(d) Field of View 1

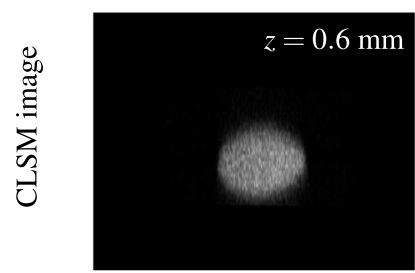

Field of View 2

Field of View 3
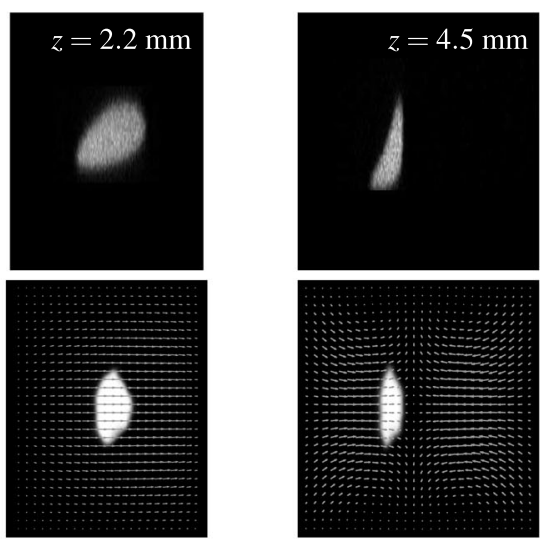

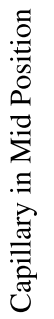
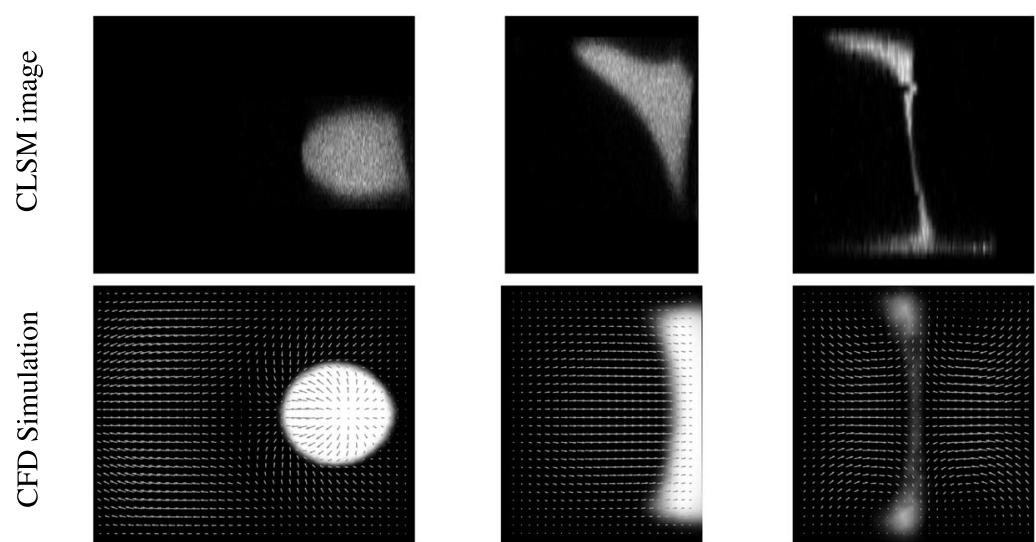

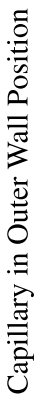

\begin{tabular}{lllllll}
\hline $\begin{array}{l}\text { Capillary } \\
\text { Position }\end{array}$ & $\begin{array}{l}\text { Flow rate } \\
\text { DI-water } \\
{[\mathrm{mL} / \mathrm{h}]}\end{array}$ & $\begin{array}{l}\text { Flow rate } \\
\text { Rhodamine B } \\
{[\mathrm{mL} / \mathrm{h}]}\end{array}$ & $\begin{array}{l}R e_{\text {channel }} \\
{[-]}\end{array}$ & $\begin{array}{l}\text { Velocity } \\
\text { Ratio } \\
{[-]}\end{array}$ & $\begin{array}{l}\text { Flow rate } \\
\text { Ratio } \\
{[-]}\end{array}$ & $\begin{array}{l}\text { Temperature } \\
\text { Range } \\
{\left[{ }^{\circ} \mathrm{C}\right]}\end{array}$ \\
\hline Mid-channel & 150 & 10 & 30 & 0.5 & 15 & $24.3<T<24.7$ \\
Mid-channel & 300 & 10 & 60 & 1.0 & 30 & $24.4<T<24.7$ \\
Mid-channel & 600 & 10 & 120 & 2.0 & 60 & $24.4<T<24.5$ \\
Near wall & 150 & 10 & 30 & 0.5 & 15 & $20.7<T<22.2$ \\
Near wall & 300 & 10 & 60 & 1.0 & 30 & $21.2<T<22.5$ \\
Near wall & 600 & 10 & 120 & 2.0 & 60 & $20.2<T<23.1$
\end{tabular}


metrics are more suitable for the model validation: The potential of diffusive mixing $\Phi$ for a segregated concentration distribution $c(x, y)$ inside any area $A_{x y}$

$\Phi\left(A_{x y}\right)=\frac{1}{\left|A_{x y}\right|} \int_{A_{x y}}\left\|\nabla\left(\frac{c}{c_{\max }}\right)\right\| \mathrm{d} A_{x y} \approx \frac{P}{A}$

is approximated as the specific contact area between two fluids [18] and can be expressed in 2D as the ratio of contact perimeter $P$ and the enclosed area $A$ of the fluid structure of a specific cross section.

The perimeter of the flow structure is determined by an image processing algorithm in MATLAB ${ }^{\circledR}$. A binary black-white image is created from the cross section data using Otsu's method to allow boundary detection and parameterization by a polygon fitting. Due to the small residence time and consequent small diffusion length, the binary image yields the fully segregated concentration distribution and allows the approximation of $\Phi$ according to the formulation of Bothe et al. [18]. Otsu's algorithm returns binary black and white pixels using a threshold based on minimizing the variance of the weighted sum of black and white values. The result of the algorithm is shown exemplary in Fig. 4c. Additionally to the potential $\Phi$, the sphericity $\Psi$ after WADELL

$\Psi=\frac{P_{\text {circle }}}{P}=\frac{\sqrt{4 \pi \cdot A}}{P}$

which relates the fluid structure perimeter $P$ to the perimeter of a perfect circle $P_{\text {circle. The sphericity gives a }}$ measure of deformation of the fluid structure and allows to compare the contribution of advection of different geometry setups.

Figure $4 \mathrm{~d}$ shows the experimental and simulation results of a selected case: FRR $=30$ with a resulting $R e_{\text {channel }}=$ 60 at room temperature. For the mid-position case, the concentration distribution is geometrically similar to the simulation results with a high reproducibility. The Rhodamine B flow structure is slightly skewed due to the geometrical focusing. However, the steady flow profile of the channel center allows only minor deformation. Some deviations between experiment and simulation can be seen which can be accounted mainly to the ideal symmetry in the simulation that can not be achieved in the experimental setup. When the capillary is placed at the outer wall, the Rhodamine B flow structure is drawn into DEAN vortices due to the inertial forces in the channel bend. The large velocity gradients near the wall cause the structure to deform by means of advection. The flow structure in the experiment slightly leans towards the channel top, whereas the CFD simulation shows ideal symmetry. Gravitational forces have a neglegible effect for the Rhodamine B is highly diluted, and the observed effect is accounted to the non-ideal symmetry in the experiment. The DEAN vortices are visualized in the CFD cross sections by means of radial velocity vectors.

The quantitative analysis of the above described case is plotted in Fig. 5. The sphericity $\Psi$ and the potential for diffusive mixing $\Phi$ of the experimental data are compared to the CFD simulation of the water-Rhodamine B system. In general, the sphericity of the initial circular flow structure $(\Psi \approx 1)$ decreases in the channel bend. With the capillary in the channel center, the decrease amounts to 0.74 in the simulation and around $10 \%$ lower in the experiment due to the previously mentioned slight asymmetry. The deformation is larger when placing the capillary near the wall. The simulation suggests a sphericity below 0.4 , and the experiment confirms this value inside the confidence bounds. The sensitivity of the deformation to the initial conditions (i.e. capillary position) is noticeable in this case, which can be seen by the large confidence bounds. This is, by definition, a strong indicator that the near wall region in the bend is subject to chaotic advection.

As expected, the look at the potential for diffusive mixing $\Phi$ presents the inverse result. The contact area of the two fluids is initially small, in the bend the potential increases close to linearly when the capillary is placed in the center. For the near wall injection, the potential grows even stronger for it is subjected to secondary flows. However, the wall contact of the Rhodamine B solution before joining the second inlet stream reduces the contact area and thus the potential for diffusive mixing for $z<3 \mathrm{~mm}$. The experimental results show good agreement with the simulation. It is therefore argued that a valid concentration distribution inherently postulates a valid velocity field as well.

\section{Reactive Mixing}

The esterification of isocyanate is simulated in the SAR mixer, as shown in Fig. 7a. The geometry is provided by the company Ehrfeld Mikrotechnik GmbH. As with the mixing Tee, the modified inlet (see Fig. 2) allows injection of small volume flows into the main feed stream. The fluids in this simulation are a di-isocyanate $\mathrm{R}_{1}-(\mathrm{NCO})_{2}$ and an alcohol $\mathrm{R}_{2}-\mathrm{OH}$ ). Figure 6 shows the concentration and velocity profile on a horizontal line in the SAR structure $(z=4.5$ mm, reference to Fig. 7) at the concentration maximum. The channel has a width of $1.5 \mathrm{~mm}$ at this cross section. The importance of resolving the grid to $\lambda_{\text {conc }}$ is shown in the graph. While the base mesh (no refinement) was constructed for an already converged momentum equation, trying to coarsen the grid in order to save computational effort heavily impacts the species solution. As defined in (6), the grid resolution is refined to $\lambda_{\text {conc }}$ to obtain a grid independent species solution. 
Fig. 5 Sphericity $\Psi$ and potential for diffusive mixing $\Phi$ of fluid structure at selected cross sections for FRR $=30$ depending on capillary position

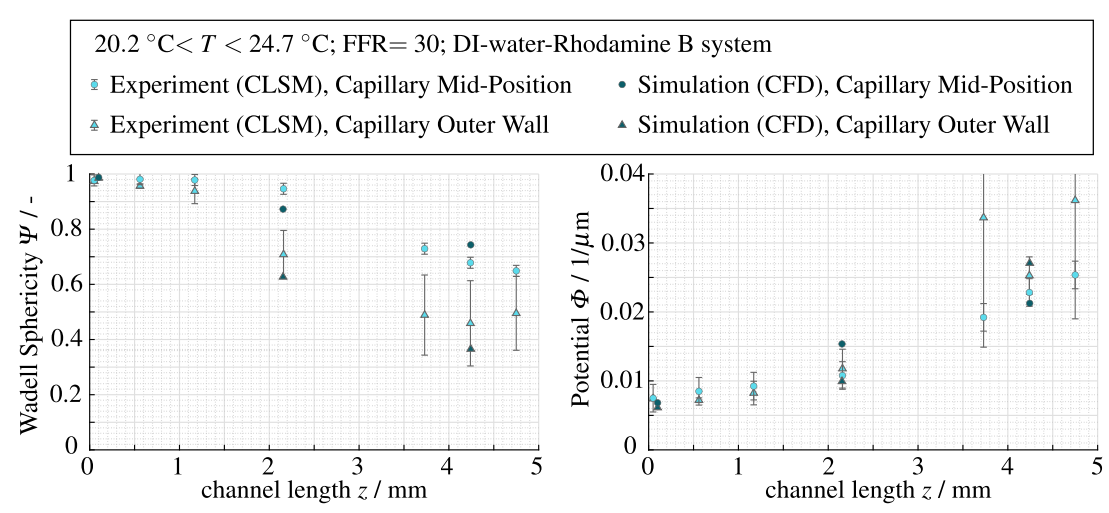

The first esterification of the di-isocyanate follows the scheme

$$
\begin{aligned}
& \mathrm{OCN}-\mathrm{R}_{1}-\mathrm{NCO}+\mathrm{HO}-\mathrm{R}_{2} \stackrel{\mathrm{k}_{1}}{\longrightarrow} \\
& \mathrm{OCN}-\mathrm{R}_{1}-\mathrm{NHCOO}-\mathrm{R}_{2}
\end{aligned}
$$

where the first cyanate group forms a carbamate (urethane) link with the alcohol. The product is termed mono-urethane for simplicity. The slower second esterification reaction uses the alcohol to form a second carbamate link

$$
\begin{aligned}
& \mathrm{R}_{2}-\mathrm{OOCHN}-\mathrm{R}_{1}-\mathrm{NCO}+\mathrm{HO}-R_{2} \stackrel{k_{2}}{\longrightarrow} \\
& \mathrm{R}_{2}-\mathrm{OOCHN}-\mathrm{R}_{1}-\mathrm{NHCOO}-\mathrm{R}_{2}
\end{aligned}
$$

yielding a di-urethane molecule. Equations (11) and (12) follow the competitive-consecutive reaction scheme of Eq. (5). The FOURIER number (4) is $F o \ll 1$, suggesting that the mixing time is smaller than the residence time. Therefore, enhanced micro mixing effects due to the different injection positions can affect the yield and the selectivity of both reactions (11) and (12).

Before analyzing the reaction, the mixing behavior of the non-reactive di-isocyanate-alcohol system is discussed. The mixing quality $\alpha$ (8), the potential for diffusive mixing $\Phi$ (9) and the WADELL sphericity $\Psi$ (10) are computed for the non-reactive simulation only. The reactive simulation is discussed subsequently. Figure $7 \mathrm{~b}$ compares the concentration distribution of the different capillary positions within the SAR mixer obtained by the nonreactive CFD simulation. The corresponding metrics (refer to Eqs. (10) and (9)) are presented in Fig. 7c. Similar to the flow pattern in the mixing Tee, the structure is skewed by the focusing of the channel in the bend for capillary mid-channel and inner wall positions when $z \leq$ $2.3 \mathrm{~mm}$. The alcohol flow structure of the capillary outer wall position follows the fluid momentum into the DEAN vortices and experiences a strong vertical stretch. This can also be noted in the decreasing sphericity. When entering the SAR structure $(z \geq 4.5 \mathrm{~mm})$, a number of effects can be observed. The helical flow configuration of the rearrange step (see Fig. 1b) leads to an engulfment-like pattern that further stretches the fluid structure for the capillary positions in the mid-channel and at the outer wall position. When the alcohol is injected near the inner wall, the structure does not separate from the wall and consequently will perform unsatisfactorily in terms of mixing and residence time distribution. The serial lamination effect of the SAR mixer is observed at $z \geq 6.9 \mathrm{~mm}$ and continues to dominate throughout the rest of the mixer length. The
Fig. 6 Non-reactive CFD simulation: Concentration profile and velocity profile on a horizontal line within the SAR structure at $z=4.5 \mathrm{~mm}$. Capillary is placed in mid-channel position
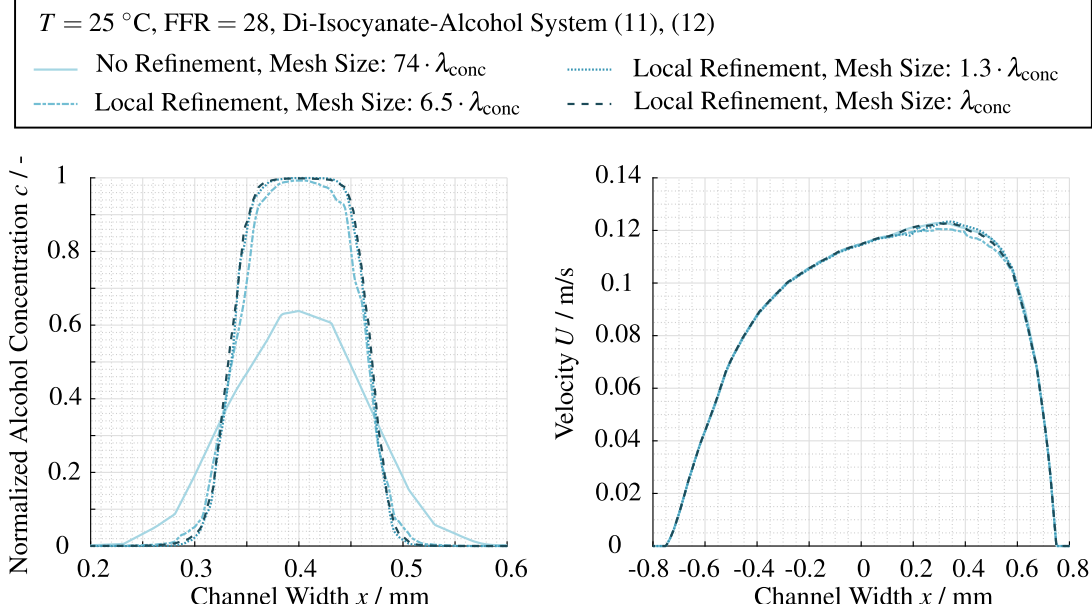

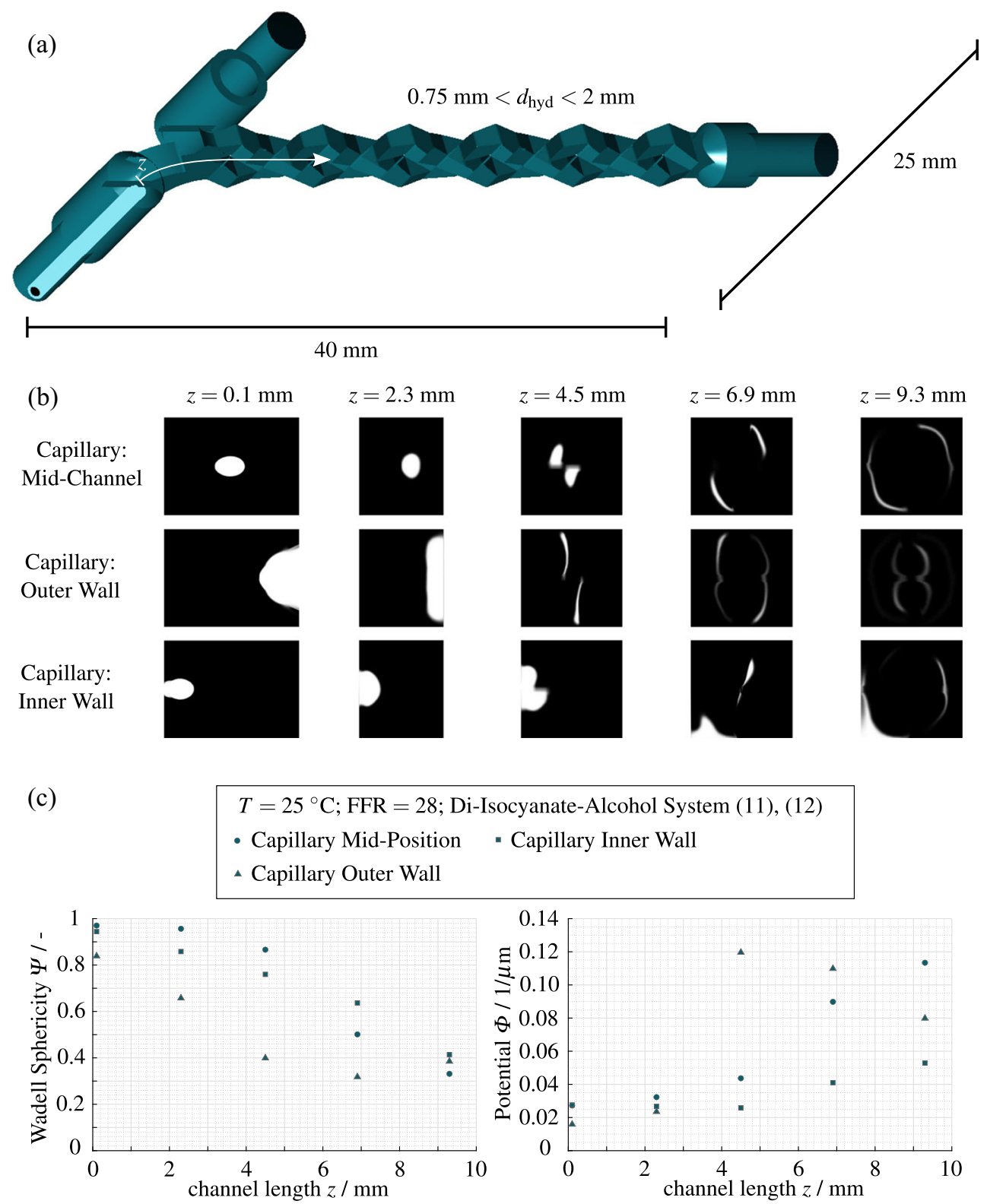

Fig. 7 Non-reactive CFD simulation: a SAR mixer geometry with modified inlet; b Alcohol (white) concentration distribution at various cross sections. $z=0 \mathrm{~mm}$ corresponds to capillary outlet; c Sphericity $\Psi$ and Potential $\Phi$ of alcohol flow structure vs channel length $z$

increased mixing performance of the capillary outer wall position is reflected in Fig. 8. The universal mixing criterion of $\alpha>0.95$ is reached at $z=16.5 \mathrm{~mm}$, whereas the other configurations achieve a mixing length of $z>23 \mathrm{~mm}$.

The reactive simulation is validated by a linear 1D kinetic model provided by the Ruhr University Bochum $(R U B)$, which assumes ideal mixing and is purely based on the kinetics. The reactions are evaluated by means of the product selectivity $S_{i}$ and the yield $Y_{i}$. Throughout the SAR mixer in all configurations, the product of the first reaction mono-urethane $C$ is preferred, Selectivity $S_{\mathrm{C}} \approx 1$, coinciding with the $1 \mathrm{D}$ model. The yield of the subsequent product di-urethane $Y_{\mathrm{D}}$ is up to four times larger in the CFD simulation compared to the $1 \mathrm{D}$ model. It suggests that the micro mixing is incomplete: Some regions exist where there is no di-isocyanate present to consume the alcohol and thus the slower reaction can take place. When comparing the capillary positions, a high potential of diffusive mixing $\Phi$ at the beginning of the channel corresponds to a larger yield of both products. The mono-urethane selectivity $S_{\mathrm{C}}$ of the capillary outer wall position starts to slightly decrease after a channel length of $z>10 \mathrm{~mm}$. However, it must be noted that the differences in selectivity are very small and are in the order of magnitude of numerical error; the total yield at 
Fig. 8 Non-reactive mixing quality $\alpha$ (refer to Eq. (8)), Mono-Urethane selectivity $S_{\mathrm{C}}$, and Di-Urethane yield $Y_{\mathrm{D}}$ over SAR mixer length. $T=25^{\circ} \mathrm{C}$, $\mathrm{FRR}=28, \operatorname{Re}=40$

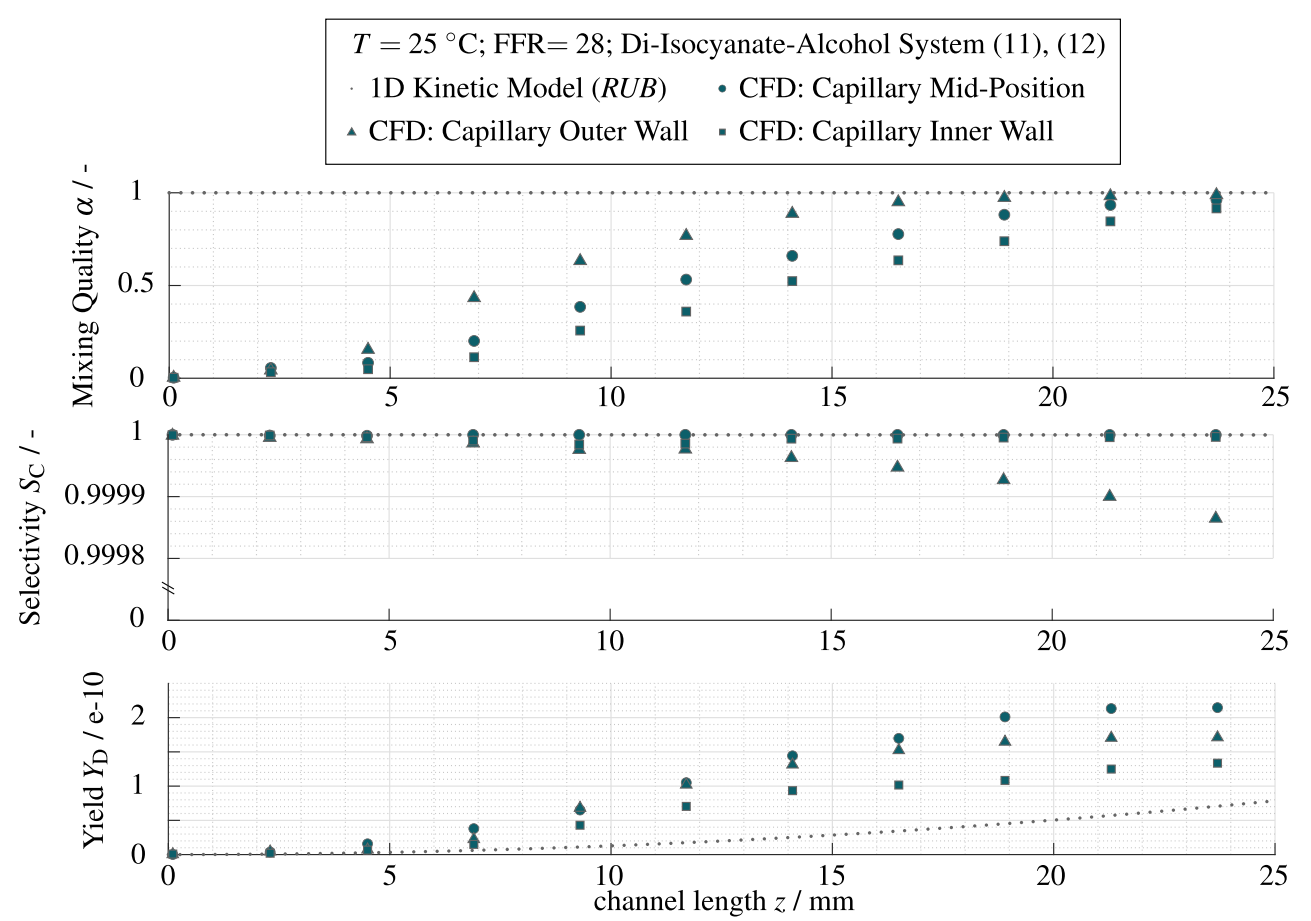

the mixer outlet is $Y_{\mathrm{C}}+Y_{\mathrm{D}}<1 e^{-3}$. According to the theory, good mixing will shift the selectivity towards the result of the $1 \mathrm{D}$ model, $S_{\mathrm{C}}=1$.

\section{Conclusion and Outlook}

The combination of Confocal Laser Scanning Microscopy using Laser Induced Fluorescence (CLSM-LIF) and CFD simulation yields reliable information about concentration distributions within a micro mixing device at low REYNOLDS numbers $(30<\operatorname{Re}<120)$. The time-independent simulation is locally refined to the smallest concentration length scale $\lambda_{\text {conc }}$ to avoid the use of micro mixing models. The mixers' inlets were adapted to facilitate flow rate ratios (FRR) between 15 and 60 without large velocity ratios. The validation of the concentration field implies a valid velocity field as well.

The results reveal the influence of the injection position on the micro mixing within the channel. Exploitation of radial secondary structures, such as DEAN vortices, is beneficial to creating a high potential of diffusive mixing and thus reducing the mixing length. In regions of secondary structures, the concentration distribution is highly sensitive to the initial conditions and indicates the presence of chaotic advection. In the inlet geometry used in this work, the best capillary position in terms of mixing was found to be at the outer channel wall.

A reactive simulation was carried out and agrees with the 1D kinetic model. However, the reaction used in this work is very slow and therefore does not give reliable information about product selectivity. The results are overlaid with numerical error. To optimize the geometry in order to reduce fouling, a test reaction, such as the fourth BOURNE or the VILLERMAUX-DUSHMAN reaction, could be more suitable.

The CLSM-LIF helps to validate and understand the complex interplay of micro mixing, residence time, and product selectivity. By using the CFD model, regions of incomplete mixing can be identified where the fluid viscosity increases due to a competitive-consecutive reaction ultimately leading to fouling. In future work, the SAR mixer will be investigated using the CLSM-LIF method. An experimental validation of the reactive flow within the SAR mixer is planned by using the VILLERMAUX-DUSHMAN test reaction. Further numerical simulations concerning the VILLERMAUX-DUSHMAN reaction are planned as well.

Supplementary Information The online version contains supplementary material available at https://doi.org/10.1007/s41981-021-00178-x.

Acknowledgements The authors gratefully acknowledge the support by the Federal Ministry for Economic Affairs and Energy (BMWi) within the ENPRO 2.0 ("Energy Efficiency and Process Intensification for the Chemical Industry", second funding period) - initiative, project "Continuous polymerisation in modular, intelligent reactors resistant to the formation of deposits (KoPPonA 2.0); sub-project: CFD modelling of deposit formation processes", grant number 03EN2004H. This project is supported by the Federal Ministry for Economic Affairs and Energy on the basis of a decision by the German Bundestag. The authors would like to express their thanks to the continued support and collaboration of the project partners COVESTRO Deutschland $A G$ and Ehrfeld Mikrotechnik GmbH. 
Funding Open Access funding enabled and organized by Projekt DEAL.

\section{Declarations}

Conflict of Interests The authors declare that they have no conflict of interest.

Open Access This article is licensed under a Creative Commons Attribution 4.0 International License, which permits use, sharing, adaptation, distribution and reproduction in any medium or format, as long as you give appropriate credit to the original author(s) and the source, provide a link to the Creative Commons licence, and indicate if changes were made. The images or other third party material in this article are included in the article's Creative Commons licence, unless indicated otherwise in a credit line to the material. If material is not included in the article's Creative Commons licence and your intended use is not permitted by statutory regulation or exceeds the permitted use, you will need to obtain permission directly from the copyright holder. To view a copy of this licence, visit http://creativecommons. org/licenses/by/4.0/.

\section{References}

1. Bayer T, Pysall D, Wachsen O (2000) Micro mixing effects in continuous radical polymerization. In: Ehrfeld W (ed) Microreaction technology: Industrial Prospects. Springer, Berlin, pp 165-170

2. Chastek TQ, Iida K, Amis EJ et al (2008) A microfluidic platform for integrated synthesis and dynamic light scattering measurement of block copolymer micelles. Lab On A Chip 8(6):950

3. Wilms D, Klos J, Frey H (2008) Microstructured reactors for polymer synthesis: A renaissance of continuous flow processes for tailor-made macromolecules? Macromol Chem Phys 209(4):343

4. Nagaki A, Takahashi Y, Akahori K et al (2012) Living anionic polymerization of tert- butyl acrylate in a flow microreactor system and its applications to the synthesis of block copolymers. Macromol React Eng 6(11):467

5. Saubern S, Nguyen X, Nguyen van et al (2017) Preparation of forced gradient copolymers using tube-in-tube continuous flow reactors. Macromol React Eng 11(5):1600065

6. Yoshida J, Kim H, Nagaki A (2017) "Impossible" chemistries based on flow and micro. J Flow Chem 7(3-4):60

7. Audus DJ, de Pablo JJ (2017) Polymer Informatics: Opportunities and Challenges. ACS Macro Lett 6(10):1078

8. Walsh DJ, Schinski DA, Schneider RA et al (2020) General route to design polymer molecular weight distributions through flow chemistry. Nat Commun 11(1):3094
9. Iwasaki T, Kawano N, Yoshida J (2006) Radical polymerization using microflow system: numbering-up of microreactors and continuous operation. Organic Process Research \& Development 10(6):1126-1131

10. Nagaki A, Nakahara Y, Furusawa M et al (2016) Feasibility study on continuous flow Controlled/Living anionic polymerization processes. Org Process Res Dev 20(7):1377

11. Nakahara Y, Furusawa M, Endo Y et al (2019) Practical Continuous-Flow Controlled/Living anionic polymerization. Chem Eng Technol 42(10):2154

12. Morsbach J, Müller AHE, Berger-Nicoletti E et al (2016) Living polymer chains with predictable molecular weight and dispersity via carbanionic polymerization in continuous flow: Mixing rate as a key parameter. Macromolecules 49(14):5043

13. Song Y, Shang M, Li G et al (2018) Influence of mixing performance on polymerization of acrylamide in capillary microreactors. AIChE J 64(5):1828

14. Hoffmann M, Schlüter M, Räbiger N (2006) Experimental investigation of liquid-liquid mixing in T-shaped micro-mixers using micro-LIF and micro-PIV. Chem Eng Sci 61(9):2968

15. Nguyen NT (2012) Micromixers: Fundamentals, design and fabrication, Micro \& nano technologies series, 2nd edn. Elsevier, Amsterdam

16. Stroock AD, Dertinger SKW, Ajdari A et al (2002) Chaotic mixer for microchannels. Science (New York, N.Y.) 295(5555):647

17. Kockmann N (2008) Transport Phenomena in Micro Process Engineering, Heat and mass transfer (Springer-Verlag, s.1.), 1. aufl. edition

18. Bothe D, Stemich C, Warnecke HJ (2004) Theoretische und experimentelle Untersuchungen der mischvorgänge in t-förmigen Mikroreaktoren- Teil 1: Numerische Simulation und Beurteilung des strömungsmischens. Chemie Ingenieur Technik 76(10):14801484

19. Reis MH, Leibfarth FA, Pitet LM (2020) Polymerizations in continuous flow: recent advances in the synthesis of diverse polymeric materials. ACS Macro Letters 9(1):123

20. DeMello AJ (2006) Control and detection of chemical reactions in microfluidic systems. Nature 442(7101):394

21. Bothe D, Stemich C, Warnecke HJ (2008) Computation of scales and quality of mixing in a T-shaped microreactor. Comput Chem Eng 32(1-2): 108

22. Guth E (1936) Untersuchungen über die Viskosität von Suspensionen und Lösungen. 1. Über die Viskosität von Suspensionen. Kolloid-Zeitschrift 74(2): 147

23. Rüttinger S, Spille C, Hoffmann M et al (2018) Laser-Induced Fluorescence in multiphase systems. Chem Bio Eng Rev 5(4):253

24. Aizinger V, Bockhorn H, Denev JA et al (2010) Macro- and Micromixing. Heat and Mass Transfer: Analysis, Simulation and Numerical Calculation, Heat and mass transfer (Springer-Verlag, s.1.), 1. aufl. edition

Publisher's note Springer Nature remains neutral with regard to jurisdictional claims in published maps and institutional affiliations. 